\title{
SELÊNIO: FUNÇÕES BIOLÓGICAS \\ E EFEITOS TÓXICOS
}

\author{
Marcelo Farina \\ Centro de Ciências da Saúde. \\ Universidade Regional Integrada do Alto Uruguai e das Missões \\ Erechim - RS
}

\section{RESUMO}

Muitos trabalhos indicam o papel toxicológico de espécies orgânicas e inorgânicas de selênio, induzindo ao aparecimento de uma variedade grande de processos que levam à injúria de tecidos de animais e de humanos. Entretanto, sabe-se que o selênio é um elemento traço essencial para o homem e que a sua deficiência é a causa principal de algumas patologias de mamíferos como doenças cardíacas e musculares. Esta revisão aborda as características químicas do selênio, relacionandoas com os seus efeitos fisiológicos e toxicológicos. Considerações sobre a absorção, metabolismo e excreção de espécies de selênio também são apresentadas.

\section{ABSTRACT}

Many studies have indicated the toxicological role of organic and inorganic species of selenium, inducing the appearance of a large variety of process that cause the injury of animals and humans tissues. 
However, its well known that selenium is an essential trace element for man and its deficiency is the major cause of some mammalian pathologies like cardiac and muscular diseases. This review approach the chemical characteristics of selenium, relating them with their physiologic and toxicologic effects. Consideration about the absorption, metabolism and excretion of species of selenium are also showed.

\section{1. - HISTÓRICO:}

Durante o século XIII, Marco Polo relatou a intoxicação de cavalos na região de Succuir, no Oeste da China. Estes animais apresentavam perda de cascos e pêlos após a ingestão de certas plantas venenosas. Seis séculos após os relatos de Marco Polo, os mesmos sintomas foram relatados em animais de pastagem que se alimentaram da vegetação nativa próxima ao Rio Missouri, entre o Sul de Dakota e Nebraska. A descoberta do agente etiológico destes efeitos tóxicos data do ano de 1928, quando Dr. Kurt Franke estudou estas plantas e seus grãos, concluindo que se tratava do selênio. Este elemento químico havia sido descoberto pelo químico sueco J. J. Berzelius, em 1817.

O selênio passou a ser um elemento conhecido, porém, sem utilidades fisiológicas. Apenas sua importância toxicológica era motivo de interesse, uma vez que atuava como o principal causador de envenenamentos de animais de fazenda. Estes apresentavam emagrecimento, perda de pêlos e anemia (FRANKE, 1934), resultando em enormes prejuízos para os criadores. Isto levou à investigação a respeito das fontes naturais de selênio, culminando na descoberta da existência de um grupo peculiar de plantas com a capacidade de acumular milhares de ppm deste elemento quando cresciam em solos seleniferos. Há provável relação entre estas espécies de plantas e os relatos de Marco Polo no século XIII. 
Enquanto os estudos de muitos pesquisadores enfatizavam a toxicidade do selênio, a essencialidade deste elemento na dieta foi demonstrada experimentalmente em 1957 por Klaus Schwarz. Em seus experimentos, este pesquisador mostrou que uma dieta deficiente de $\alpha$ tocoferol (vitamina E) causava necrose hepática em ratos e que o selênio era um nutriente essencial capaz de prevenir o desenvolvimento desta doença. Muitas outras desordens causadas pela deficiência de selênio foram subseqüentemente identificadas em ovelhas, gado, porcos e aves (EGGERT et al., 1957; MUTH et al., 1958; CALVERT et al., 1962; HARTLEY et al., 1961).

As concentrações extremamente baixas nas quais o selênio atuava biologicamente sugeriam um possível envolvimento enzimático deste elemento. Na década de setenta, foi demonstrado que o selênio é um componente essencial para a enzima glutationa peroxidase, estando ligado a esta de forma covalente (FLOHE et al., 1973; ROTRUCK et al., 1973), e seu papel metabólico nesta selenoproteína relaciona-se com a proteção dos componentes teciduais contra a destruição oxidativa. Mais recentemente, outras selenoenzimas como a 5'-deiodinase (BEHNE \& KYRIAKOPOULOS, 1990) e a fosfolipídio hidroperóxido glutationa peroxidase (URSINI et al., 1982) foram descobertas, apresentando papeis no metabolismo de hormônios tireoideanos e na redução de hidroperóxidos de fosfolipídios, respectivamente.

Atualmente, a deficiência de selênio tem sido apontada como a principal causa de algumas patologias do organismo humano, onde o músculo cardíaco é o tecido mais suscetível a danos. Em certas regiões da China, como a região de Keshan, onde há um baixo conteúdo de selênio no solo, existe grande incidência de um tipo específico de cardiomiopatia humana causada pela deficiência deste elemento, caracterizada pela destruição das membranas das células do músculo. cardíaco, as quais são substituídas por fibroblastos (YANG, 1987). Nesta 
patologia ocorre aumento no tamanho do coração, o qual passa ser composto predominantemente por tecido fibrótico não funcional. Outros sintomas desta cardiomiopatia, também conhecida como doença de Keshan, são: náusea, perda do apetite, vertigens, sensação de frio, eletrocardiograma anormal e falência cardiaca congestiva. As crianças que vivem nestas regiões e que não recebem suplementação de selênio nas suas dietas, geralmente desenvolvem esta cardiomiopatia. Apesar de não estarem bem esclarecidos os mecanismos pelos quais o selênio protege as células cardíacas, evitando o aparecimento da doença de Keshan, flutuações na incidência sazonal da doença sugerem o envolvimento de um agente infeccioso. Estudos de LEAVANDER \& BECK (1997) demonstram que um coxsackievirus B4, isolado a partir de vítimas desta cardiomiopatia, causou maior destruição do tecido muscular ao ser incubado em camundongos com deficiência de selênio quando comparada aos animais com níveis endógenos normais do elemento. Além da doença de Keshan, que é uma patologia predominante apenas nas regiões com baixo conteúdo de selênio no solo, outras alterações cardiacas foram observadas em indivíduos com desordens neurológicas e que alimentavam-se através de sondas. As fórmulas nutricionais utilizadas para estes pacientes eram deficientes em selênio e os níveis endógenos do elemento nestes indivíduos eram menores quando comparados aos niveis de pacientes que se alimentavam normalmente. Além disso, os pacientes que alimentavam-se através de sondas e que, consequentemente, apresentavam deficiência de selênio, demonstraram anormalidades em seus eletrocardiogramas e ecocardiografia quando comparados ao grupo controle. A suplementação destes pacientes com selênio normalizou os níveis endógenos do elemento, melhorando parcialmente as anormalidades nos seus eletrocardiogramas e aumentando as funções cardíacas observadas na ecocardiografia (SAITO et al., 1998). 
Neste contexto, a suplementação de dietas de animais e humanos com selênio tem sido aceita pela comunidade científica. Para humanos, a Junta de Alimentação e Nutrição da Academia de Ciências dos Estados Unidos propôs a ingestão diária de 50 a $200 \mu \mathrm{g}$ de selênio, considerando esta quantidade adequada e isenta de efeitos tóxicos em indivíduos adultos. Isto tem ajudado a aliviar os problemas das regiões onde as concentrações de selênio no solo são muito baixas.

Mesmo que se considere a essencialidade do selênio para humanos e o seu valor preventivo e terapêutico contra determinadas doenças, sua suplementação dietética deve ser efetuada com extremos cuidados, já que o limiar entre os níveis requeridos e os níveis tóxicos, para muitos organismos, é apenas cerca de uma ordem de magnitude, o que é realmente muito raro para um elemento traço essencial.

\section{2. - PROPRIEDADES QUÍMICAS RELACIONADAS AOS SISTEMAS BIOLÓGICOS:}

O selênio é um elemento do grupo $\mathrm{VI}$, podendo existir nos estados de oxidação $\mathrm{Se}^{6+}, \mathrm{Se}^{4+}, \mathrm{Se}^{2+}, \mathrm{Se}^{0}$ e $\mathrm{Se}^{2-}$. Compartilha propriedades físicas e químicas com o enxofre. Os raios atômicos e iônicos, a eletronegatividade e o potencial de ionização são parecidos em ambos os elementos. Esta similaridade permite que o selênio substitua 0 enxofre, promovendo interações selênio-enxofre nos sistemas biológicos. Por outro lado, as diferenças nas propriedades físico-químicas entre selênio e enxofre constituem a base de seus papéis biológicos especificos (STADTMAN, 1980).

Os selenóis (R-SeH) são as formas correspondentes dos tióis (R-SH), onde o átomo de selênio substitui o átomo de enxofre. As principais diferenças entre selenóis e tióis, relevante para os sistemas bioquímicos, estão relacionadas com suas constantes de dissociação e 
caracteres nucleofilicos. Os selenóis são ácidos mais fortes que os tióis. Os primeiros estão preferencialmente sob a forma dissociada em $\mathrm{pH}$ fisiológico, enquanto a predominância da forma tiólica é não-dissociada. Devido a esta diferença química entre tióis e selenóis, estes últimos são capazes de reduzir dissulfetos, sulfóxidos e compostos aromáticos nitrogenados (KLAYMAN \& GÜNTHER, 1973). Pelas suas oxidações, os selenóis são convertidos em seus correspondentes disselenetos. O agente oxidante pode ser o hidroperóxido (EQUAÇÃO I).

\section{$2 \mathrm{R}-\mathrm{SeH}+\mathrm{H}_{2} \mathrm{O}_{2} \rightarrow \mathrm{R}-\mathrm{SeSe}-\mathrm{R}+2 \mathrm{H}_{2} \mathrm{O}$}

Os disselenetos podem ser oxidados a ácido selenênico, selenínico ou selenônico, dependendo das condições utilizadas. Além disso, os disselenetos podem ser reduzidos a selenóis por agentes redutores como mercaptoetanol, DTT, cisteina e glutationa (KLAYMAN \& GÜNTHER, 1973).

O selenito pode oxidar tióis como cisteína, glutationa, coenzima A, ácido lipóico e cisteína protéica (GANTHER, 1971). Esta reação apresenta importância fisiológica e provavelmente constitui o primeiro passo para a incorporação do selênio inorgânico nos sistemas biológicos. A reação entre o selenito e tióis, primeiramente proposta por PAINTER (1941), foi posteriormente estudada por TSEN e TAPPEL (1958) e, de modo bastante detalhado por GANTHER (1968). Um intermediário do tipo selenotrissulfeto é formado na reação global (EQUAÇÃO II).

$$
4 \mathrm{R}-\mathrm{SH}+\mathrm{HSeO}_{3}{ }^{-}+\mathrm{H}^{+} \rightarrow \mathrm{R}-\mathrm{SSeS}-\mathrm{R}+\mathrm{R}-\mathrm{SS}-\mathrm{R}+3 \mathrm{H}_{2} \mathrm{O}
$$

Os selenotrissulfetos reagem com reagentes nucleofilicos e são várias ordens de magnitude mais reativos que os correspondentes dissulfetos. O intermediário formado pela reação entre a glutationa 
reduzida e o ácido selenoso, GSSeSG, pode, pela interação com GSH em pH fisiológico, formar selenopersulfeto (GANTHER, 1971) (EQUAÇÃO III).

\section{GSSeSG + GSH $\rightarrow$ GSSeH + GSSG}

Os selenetos orgânicos também apresentam importantes particularidades nos sistemas biológicos. O dimetil-seleneto é reconhecido como um produto volátil do metabolismo do selênio em animais, sendo formado por redução e sucessiva metilação de sais inorgânicos de selênio (HSIEH \& GANTHER, 1976). O dimetil-seleneto e outros selenetos (dialquil- e alquilarilselenetos) são bons substratos para as monooxigenases microssomais que contêm flavina (FMO), podendo, assim, serem oxidados a seus selenóxidos correspondentes (GOEGER \& GANTHER, 1994; CHEN \& ZIEGLER, 1994). Estes são potentes oxidantes de tióis e possivelmente atuem em proteínas que possuem grupamentos $-\mathrm{SH}$.

Os selenoaminoácidos são capazes de catalisar as trocas tiol-dissulfeto (DIKSON \& TAPPEL, 1969) que podem ser relevantes em sistemas biológicos. Além disso, os selenoaminoácidos podem decompor hidroperóxidos mais eficientemente que os compostos correspondentes de enxofre (CALDWELL \& TAPPEL, 1965). Esta maior reatividade pode ser atribuída ao maior caráter nucleofilico dos compostos de selênio. A reação entre hidroperóxidos e a porção selenol de selenoaminoácidos ocorre, provavelmente em dois passos seqüenciais (EQUAÇÃO IV).

\section{$\mathrm{R}-\mathrm{SeH}+\mathrm{R}-\mathrm{OOH} \rightarrow \mathrm{R}-\mathrm{SeOH}+\mathrm{R}-\mathrm{H}$ \\ $\mathrm{R}-\mathrm{SeH}+\mathrm{R}-\mathrm{SeOH} \rightarrow \mathrm{R}-\mathrm{SeSe}-\mathrm{R}+\mathrm{H}_{2} \mathrm{O}$}

Num primeiro passo, o selenol é oxidado a ácido selenênico e, num segundo passo, ocorre a formação de um disseleneto pela interação com outro selenol. Estas reações são responsáveis pela 
atividade do tipo peroxidase de selenocistina e selenocistamina (YASUDA et al., 1980).

\section{3. - SELENOPROTEÍNAS:}

As selenoproteínas já identificadas possuem o selênio na forma de selenocisteina, com exceção de uma selenoproteína de origem bacteriana, a tiolase, que apresenta selênio na forma de selenometionina. Esta última não é sintetizada por humanos e deve ser suplementada pela dieta (COWIE \& COHEN, 1957).

A selenocisteína, forma biologicamente ativa de selênio, está presente em proteínas como glutationa peroxidase (FLOHE et al., 1973; ROTRUCK et al., 1973), 5'-deiodinase (BEHNE \& KYRIAKOPOULOS, 1990), fosfolipídio hidroperóxido glutationa peroxidase (URSINI et al., 1982) e selenoproteína P (LINDER, 1990).

A primeira selenoproteína caracterizada foi a glutationa peroxidase (GSHPx), que apresenta um papel protetor contra a peroxidação de lipídios ao catalisar reações redox entre hidroperóxidos e GSH, reduzindo o peróxido de hidrogênio ou outros hidroperóxidos não ligados as membranas celulares (EQUAÇÃO V).

\section{$\mathrm{ROOH}+2 \mathrm{GSH} \rightarrow \rightarrow \rightarrow \rightarrow \rightarrow \rightarrow \rightarrow \mathrm{ROH}+\mathrm{GSSG}+\mathrm{H}_{2} \mathrm{O}$ \\ GSHPX R-alquil ou H}

A enzima fosfolipídio hidroperóxido glutationa peroxidase (PHGSHPx) apresenta função similar a GSHPx na redução de hidroperóxidos, entretanto, é capaz de catalisar apenas a redução de hidroperóxidos lipídicos nas membranas celulares. Além disso, pode utilizar outros tióis como substratos além da GSH (EQUAÇÃO VI).

$$
\mathrm{R}^{\prime} \mathrm{OOH}+2 \mathrm{RSH} \rightarrow \rightarrow \rightarrow \rightarrow \rightarrow \rightarrow \rightarrow \mathrm{R}^{\prime} \mathrm{OH}+\mathrm{RSSR}+\mathrm{H}_{2} \mathrm{O}
$$

\section{PHGSHPX R'-fosfolipídios}


A 5'-deiodinase é uma selenoproteína de membrana que catalisa a conversão do hormônio L-tiroxina $\left(T_{4}\right)$ para o hormônio biologicamente ativo 3,3'-5 triodotironina $\left(T_{3}\right)$. Esta enzima já foi encontrada em tireóide, fígado e rins de ratos. Estudos com selênio marcado ( $\left[{ }^{75} \mathrm{Se}\right.$-selenito) demonstraram que as subunidades da 5 'deiodinase contém um átomo de selênio por monômero e que a selenocisteína é um resíduo essencial para a catálise desta enzima (BEHNE \& KYRIAKOPOULOS, 1990).

A selenoproteína $P$, outra proteína que contém selenocisteína, está presente no plasma de mamíferos e apresenta uma rara composição, contendo no mínimo 8 resíduos de selenocisteína por subunidade. Suas funções ainda não estão bem esclarecidas, entretanto, sugere-se que sua função seja transportar selênio do fígado para os testículos (LINDER, 1990).

A inserção altamente específica da selenocisteína nas enzimas dependentes de selênio é um assunto que não está completamente elucidado, proporcionando uma excitante área de pesquisa sobre a bioquímica do selênio. Um dado muito importante a partir das observações de SUNDE (1987) indica que o esqueleto carbônico do resíduo de selenocisteína na glutationa peroxidase é derivado diretamente do aminoácido serina. Pouco tempo depois, foi descoberto que o gene que codifica esta enzima contém uma trinca de bases "TGA" na posição correspondente a selenocisteína (GÜNZLER et al., 1984). Em eucariontes, ocorre o envolvimento de um O-fosfoseril como um intermediário no processo de conversão de Seril-tRNA ${ }^{\mathrm{Sec}}$ em Selenocisteinil-tRNA ${ }^{\text {Sec }}$ (EQUAÇÃO VII).

\section{ATP RSe}

Seril-tRNA ${ }^{\text {Sec }} \rightarrow \rightarrow \rightarrow \rightarrow O$-fosfoseril-tRNA ${ }^{\text {Sec }} \rightarrow \rightarrow \rightarrow$ Selenocisteinil-tRNA $^{\text {Sec }}$

\section{Quinase}


A selenocisteína resultante desta combinação (Se-cystRNA) reconhece o códon "UGA" (LINDER, 1990).

\section{4. - ABSORÇÃO, DISTRIBUIÇÃO E EXCREÇÃO:}

$A$ absorção de selênio sob a forma de selenito através do trato gastrointestinal de ratos excede $90 \%$ (BROWN et al., 1972). Este valor é elevado quando comparado à absorção intestinal de selenito em humanos (59\%) (THOMSON \& STEWART, 1974). Os selenoaminoácidos tais como selenometionina são bem absorvidos por esta via tanto por ratos quanto por humanos (90\%) (THOMSON \& STEWART, 1973). A partir de estudos em ratos usando ${ }^{75} \mathrm{Se}$ sob a forma de selenito e de selenometionina, a absorção a nivel de estômago foi nula. O principal local de absorção parece ser o duodeno, seguido pelo jejuno e íleo. Além do trato gastrointestinal, o selênio pode ser absorvido por tecidos cutâneos ou por inalação. Estas duas últimas vias de absorção de selênio estão relacionadas com a exposição e intoxicação ocupacional por compostos de selênio (WHANGER et al., 1976).

O selênio pode ser transportado do intestino para o fígado principalmente unido a VLDLs e LDLs. A albumina e a $\alpha$-globulina apresentam importante papel no transporte de selênio através do sistema circulatório (CAVALIERI, 1980).

A primeira evidência de que compostos de selênio são metabolizados em animais foi determinada após um longo período de tratamento com selenito de sódio. Os animais apresentavam um odor gárlico característico, que foi posteriormente demonstrado ser causado pelo dimetil-seleneto (KLAYMAN \& GUNTHER, 1973). Este composto pode ser exalado e é resultado da detoxificação metabólica de muitos compostos de selênio, a qual envolve uma série de metilações dependentes da S-adenosilmetionina (HOFFMAN \& McCONNELL, 1986). 
Atualmente sabe-se que tanto os selenoaminoácidos quanto os compostos inorgânicos de selênio são metabolizados em animais, sendo que a principal via metabólica é a mesma para ambas as classes, envolvendo a interação com tióis, conforme mostra a FIGURA 1. A distribuição e o acúmulo de selênio varia com o tipo de tecido, o nível de selênio, o tipo de composto e a suscetibilidade do organismo. Em animais intoxicados cronicamente, o selênio é depositado principalmente nos rins e fígado, seguido pelo pâncreas, baço e pulmões (WILBER, 1980). Poucas informações estão disponiveis em relação a distribuição de selênio em tecidos humanos após a administração de ${ }^{75}$ Se. No entanto, existem dados sugerindo que as concentrações mais elevadas encontram-se nos rins e fígado, como foi observado em ratos, e baixos niveis podem ocorrer no sangue, pulmões, baço, pâncreas, coração e testículos (CAVALIERI et al., 1966).

O selênio pode ser eliminado do organismo pelas três principais vias excretoras - urina, fezes e ar expelido. Os rins têm um importante papel na homeostase do selênio, pois a excreção urinária é considerada uma das principais rotas de desintoxicação e de eliminação do elemento em animais e humanos (BOOP et al., 1982). O selênio excretado na urina pode ser usado como indicador em casos de intoxicação ou de exposição a niveis altos do elemento (VALENTINE et al., 1978). Medidas da concentração de selênio na urina têm sido usadas amplamente no diagnóstico de algumas doenças como cardiomiopatias (GOLDMAN \& KANTROWITZ, 1981), câncer, epilepsia (HOJO,1981) e queimaduras (HUNT et al.,1983).

Mesmo que a excreção urinária de selênio tenha importância primária na eliminação do elemento por muitos animais, quantidades apreciáveis são eliminadas pelas fezes, mesmo após administração parenteral. A excreção fecal representou cerca de $10 \%$ da quantidade de selênio administrada intraperitonialmente 


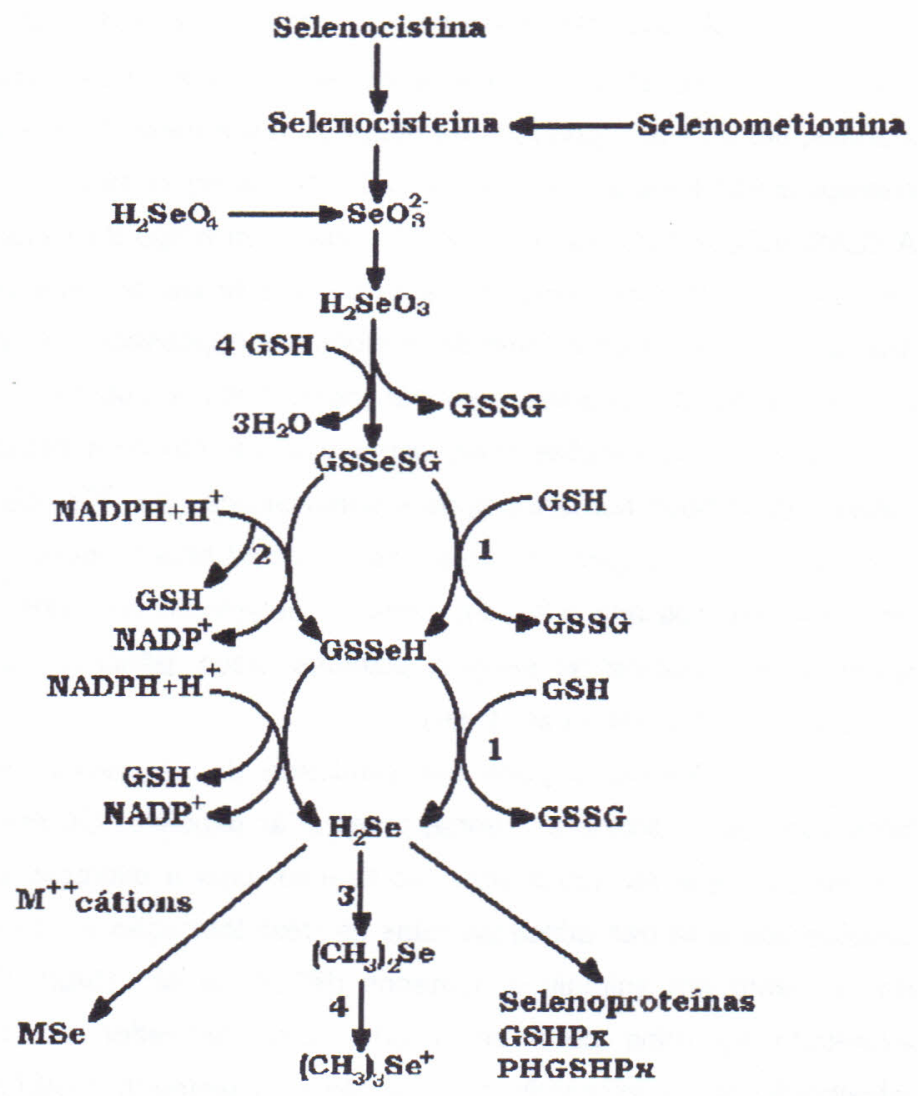

Figura 1. Metabolismo de compostos inorgânicos e orgânicos de selênio em animais.

1. Reação não-enzimática $\quad 3$. Seleneto metiltransferase

2. Glutationa redutase 4. Dimetilseleneto metiltransferase

(HOPKINS et al., 1966). Tem sido demonstrado que a excreção biliar pode contribuir, mesmo que em pequena escala, para a eliminação de selênio, e que esta via pode ser favorecida quando compostos de arsênio são administrados com selênio (LEVANDER \& BAUMANN, 1966).

70 Rev. Ciência e Natura, Santa Maria, 22: 59 - 81 , 2000. 
Informações sobre a excreção fecal de selênio em humanos são raras. THOMSON \& STEWATR (1974) relataram niveis altos de selênio nas fezes de sujeitos que receberam ${ }^{75}$ Se-selenito por via oral, variando de 33 a $58 \%$ da dose. Quando o mesmo composto foi administrado pela via endovenosa, a excreção fecal foi mínima $(<1 \%)$ (KUIKKA \& NORDMAN, 1978).

A excreção de selênio pelo ar expirado é feita basicamente pelo composto volátil dimetil-seleneto. $\mathrm{O}$ odor gárlico, característico deste composto, tem sido detectado na respiração de indivíduos expostos acidentalmente a níveis altos de selênio (MOZIER et al., 1988).

\section{5. - TOXICIDADE:}

Os efeitos tóxicos do selênio foram primeiramente observados em animais, os quais eram intoxicados após a ingestão de certas espécies de grãos e plantas (FRANKE, 1934). Dois tipos de selenoses foram definidas: a doença alcalina e o falso cambalear. A doença alcalina ("alkali desease") é caracterizada por retardo no crescimento, deformações nos cascos, perda de pêlos e, eventualmente, morte. Esta toxicidade crônica resulta da ingestão de grãos e plantas, onde o selênio está presente na forma de selenoaminoácidos de proteínas (selenocisteina e selenometionina) (FRANKE, 1934; PAINTER \& FRANKE, 1936). O falso cambalear ("blind staggers") é uma desordem de caráter mais agudo, comprometendo, predominantemente, o sistema nervoso central. Nesta condição, os animais tropeçam, chocam-se contra objetos e podem apresentar paralisia seguida de morte. Esta doença resulta do consumo de certas plantas "acumuladoras" de selênio como as espécies do gênero Astragalus que podem conter concentrações do metal na ordem de milhares de ppm (MARTIN \& GERLACK, 1972) sob a forma 
de selenoaminoácidos do tipo não protéico como a selenocistationina e a selenometilselenocistina (TRELEASE et al., 1960; SHRIFT et al., 1965).

Atualmente, sabe-se que o selênio não apresenta efeito tóxico apenas para animais de pastagem. O homem também é suscetível a intoxicação por selênio, principalmente pelo seu amplo uso na indústria. Uma variedade de compostos de selênio são usados em retificadores, semicondutores, células fotoelétricas, eletrodos, câmeras, televisões, baterias solares, cabos elétricos, na vulcanização da borracha, na fabricação de plásticos, na manufatura do ácido sulfúrico, na síntese de compostos orgânicos e no processamento da pirita (WILBER, 1980). Alguns compostos de selênio são usados como oxidantes na preparação da niacina e da cortisona, como antioxidantes em óleos de lubrificação e como agentes de coloração em fotografias, tintas, vidros, e pinturas (CARTER, 1966). Industrialmente, o selênio também é encontrado nos sedimentos que resultam do processo de refinamento do cobre por eletrólise. Esses sedimentos apresentam $7 \%$ de selênio em sua composição; niveis estes considerados acima do recomendado pela "American Conference of Governmental Industrial Hygienist" (HOLNES et al., 1989). Apesar da utilização de agentes de proteção contra acidentes de trabalho (luvas, roupas protetoras e ventilação adequada) alguns casos de dermatite aguda, queimaduras de pele, edema pulmonar e respostas alérgicas foram observados em trabalhadores de indústrias expostos ao $\mathrm{SeO}_{2}$ (AMOR \& PRINGLE, 1945). A intoxicação ocupacional crônica por selênio pode ocasionar outros efeitos como gosto metálico na boca, odor de alho na respiração, irritação das mucosas, gastroenterites, pigmentação avermelhada em unhas, cabelos e dentes (BUCHAN, 1947; CERWENKA \& COOPER, 1961). Em casos de exposição a longo prazo, os órgãos mais afetados pela toxicidade do selênio são os do sistema pulmonar seguidos pelos órgãos do sistema gastrointestinal e os do sistema nervoso central (DISKIN et al., 1979). 
A intoxicação humana por selênio também pode estar relacionada com a alimentação. $A$ ingestão de cereais e vegetais de uma área da República da China com altos níveis deste elemento no solo resultou em sintomas como perda de cabelos e unhas, lesões de pele, parestesia e polineurite (YANG et al., 1983). Outro relato de pessoas expostas a áreas e grãos seleníferos mostra o desenvolvimento de disfunções gástricas, intestinais e hepáticas (SMITH et al., 1936). Nos Estados Unidos também foram constatados casos de intoxicação humana por selênio devido ao consumo de alimentos com altas concentrações do elemento. Os sintomas consistiam em vômitos, irritabilidade, fadiga e neuropatia periférica (HELZLSOUER et al., 1985).

Alguns fatores são considerados protetores na toxicologia do selênio por diminuírem a toxicidade provocada pelo mesmo. Dentre eles está o arsênio que aumenta a excreção biliar de selênio e a metionina + vitamina $\mathrm{E}$ que protegem o tecido hepático de necroses causadas por selenoses crônicas (OKAMOTO \& GÜNTHER, 1972; WILBER, 1980).

Sabe-se que o selênio proveniente de diferentes fontes não é igualmente tóxico. Isto é válido porque os diferentes tipos de compostos de selênio apresentam distintas características físicas e químicas, as quais refletem suas capacidades de interação com moléculas endógenas, permeabilidade celular e solubilidade (BARBOSA et al, 1998).

\section{6. - MECANISMOS DE DANOS BIOLÓGICOS:}

Desde a descoberta de que o selênio possui propriedades tóxicas em relação a muitos animais, numerosas hipóteses tentaram explicar seu mecanismo de toxicidade. Atualmente, apesar do rápido progresso no entendimento do metabolismo do selênio, os mecanismos. de sua toxicidade ainda não estão bem definidos. 
Em 1941, PAINTER foi o primeiro a propor que a toxicidade do selênio (selenito) era devido a sua interação com tióis. As reações entre compostos de selênio e tióis foram posteriormente estudadas por TSEN \& TAPPEL (1958) e, de modo bastante detalhado por GANTHER (1968), que sugeriu a interação do selênio com tióis endógenos, formando selenotrissulfetos (ver equação II). Estes, apesar de possuírem relativa estabilidade, podem ser reduzidos com a formação de selenopersulfetos (ver equação III).

Outro grande avanço a respeito da toxicidade do selênio ocorreu no final da década de oitenta, quando foi proposta a formação de superóxido $\left(\mathrm{O}_{2}{ }^{*-}\right)$ proveniente da reação de selenito com glutationa (SEKO et al., 1989) (EQUAÇÃO VIII).

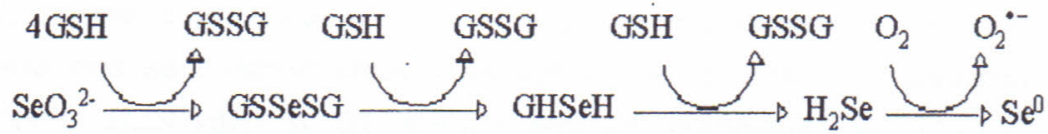

Experimentos de SPALLHOLZ (1993) demonstraram que o selenito e a selenocistina possuem a propriedade de causar dano em membranas eritrocitárias in vitro. Neste experimento, houve um declínio da GSH celular como uma conseqüência da citotoxicidade do selênio e hemólise. A formação de radicais livres foi apontada como a responsável pela injúria celular. Além do superóxido, as reações entre selenito e GSH foram capazes de produzir $\mathrm{H}_{2} \mathrm{O}_{2}$ (YAN \& SPALLHOLZ, 1991).

Estudos usando hepatócitos isolados de ratos tratados com selenito mostram o possivel envolvimento de espécies reativas de oxigênio como causa da ação citotóxica do selênio. Demonstrou-se que nestas células ocorre um aumento na taxa de consumo de oxigênio (ANUNDI et al., 1984; KITAHARA et al., 1993) e na liberação da enzima 
lactato desidrogenase (LDH) (KITAHARA et al., 1993), e uma redução nos niveis de GSH intracelular (ANUNDI et al., 1984; GARBERG et al., 1988) e na lipoperoxidação (KITAHARA et al., 1993). Formas orgânicas de selênio também podem facilitar a oxidação de grupos $-\mathrm{SH}$ de moléculas de baixo peso molecular como a tiocolina (SCHONEICH et al., 1990; GOEGER \& GANTHER, 1994).

Recentemente, BARBOSA e colaboradores demonstraram que compostos orgânicos de selênio são capazes de inibir a enzima sulfidrílica $\delta$-aminolevulinato desidratase ( $\delta$-ALA-D). Esta enzima, que catalisa uma das reações da síntese do heme, é inibida por estes compostos pela oxidação de grupos $-\mathrm{SH}$ presentes no seu sítio catalítico.

Determinados compostos orgânicos de selênio (dialquil e alquilarilselenetos) podem ser oxidados aos seus respectivos selenóxidos, os quais são considerados potentes oxidantes tiólicos. A oxidação de selenetos em selenóxidos pode ser catalisada por monoxigenases microssomais. As FMO (monoxigenases contendo flavina) podem aumentar a oxidação de selenetos a selenóxidos na presença de NADPH e oxigênio, assim, aumentando as propriedades oxidantes e toxicológicas destas espécies orgânicas de selênio (CHEN \& ZIEGLER, 1994). 


\section{7. -REFERÊNCIAS BIBLIOGRÁFICAS:}

AMOR, A. J. \& PRINGLE, P. A review of selenium as an industrial hazard. Bull. Hyg. 20: 239 (1945).

ANUNDI, I.; STAHL, A.; HOGBERG, J. Effects of selenite on $\mathrm{O}_{2}$ consumption, glutathione oxidation and NADPH levels in isolated hepatocytes and the role of redox changes in selenite toxicity. Chem. Biol. Interact. 50: 277-288 (1984).

BARBOSA, N. B. V.; ROCHA, J. B. T.; ZENI, G.; EMANUELLI, T.; BEQUE, M. C.; BRAGA, A. L. Effect of organic forms of selenium on $\delta$ aminolevulinate dehydratase from liver, kidney, and brain of adult rats. Toxicol. Appl. Pharmacol. 149: 243-253 (1998).

BEHNE, D.; KYRIAKOPOULOS, A. Identification of type I iodothyronine 5'deiodinase as a selenoenzyme. Biochem. Biophys. Res. Commun 173: $1143-1149$ (1990).

BOOP, B. A.; SONDERS, R. C.; KESTERSON, J. W. Metabolic fate of selected selenium compounds in laboratory animals and man. Drug. Metabolism. Review. 13 (2): 271-318 (1982).

BROWN, D. G.; BURK, R. F.; SEELY, R. J.; KIKER, K. W. Effects of dietary selenium on the gastrointestinal absorption of $(75 \mathrm{Se})_{2}$ in rat. Int. J. Vitam. Nutr. Res. 42: 588-591 (1972).

BUCHAN, R. F. Industrial selenosis. Occupational Medicine. 3: 439 (1947).

BUEL, D. N. Potential hazards of selenium as a chemopreventive agent. Seminars in Oncology. 10: 311-321 (1983).

CALDWELL, K. A. \& TAPPEL, A. L. Acceleration of sulphydryl oxidations by selenocystine. Arch. Biochem. Biophys. 112: 196-200 (1965).

CALVERT, C. C.; NESHEIM, M. C.; SCOTT, M. L. Effectiveness of selenium in prevention of nutritional muscular dystrophy in the chick. Proc. Soc. Exp. Biol. Med. 109: 16-18 (1962).

CARTER, R. F. Acute selenium poisoning. The Medical Journal of Australia. 26: 525-527 (1966).

CAVALIERI, R. R. In Goodhart, R. S.; Shils, M. E., eds: Modern nutrition in health and disease $\left(6^{\text {th }}\right.$ ed). Philadelphia: Lea \& Febiger, 395pp. (1980).

CAVALIERI, R. R.; SCOTT K. G.; SAIRENJI, E. Selenite (75Se) as a tumor-localizing agent in man. J. Nucl. Med. 7: 197-208 (1966).

CERWENKA, E. A. \& COOPER, W. C. Toxicology of selenium and tellurium compounds. Arch. Environ. Health. 3: 189-199 (1961).

CHEN, G. \& ZIEGLER, D.M. Liver microsomes and flavin-containing monooxygenase catalyzed oxidation of organic selenium compounds. Arch. Biochem. Biophys 312: 566-572 (1994). 
COWIE, D. B. \& CHOEN, G. N. Biosynthesis by Escherichia coli of altered proteins containing selenium instead of sulfur. Biochim. Biophys. Acta. 26: 252-261 (1957).

DIKSON, R. C. \& TAPPEL, A. L. Effects of selenocystine and selenomethionine on activation of sulphydryl enzimes. Arch. Biochem. Biophys. 131: 100-110 (1969).

DISKIN, C. J.; TOMASSO, C. L.; ALPER, J. C.; GLASER, M. L.; FIEGEL, S. E. Long-term selenium exposure. Arch. Intern. Med. 139: 824-826 (1979).

EGGERT, R. G.; PATTERSON, E.; AKERS, W. T.; STOKSTAD, E. L. R. The role of vitamin $E$ and selenium in the nutrition of the pig. J. Anim. Sci. 16: 1037 (1957).

FLOHE, L.; GUNZLER, W. A.; SCHOCK, H.H. Glutathione peroxidase: A selenium enzyme. FEBS Lett 32: 132-134 (1973).

FRANKE, K. W. A new toxicant occurring naturally in certain samples of plant foodstuffs. I. Results obtained in preliminary feeding trials. $\mathrm{J}$. Nutr. 8: 597-608 (1934).

GANTHER, H. E . Selenotrisulfides. Formation by reaction of thiols with selenious acid. Biochemistry. 10: 4089-4098 (1971).

GANTHER, H.E. Selenotrisulfides. Formation by reaction of thiols with selenious acid. Biochemistry. 7: 2898-2905 (1968).

GARBERG, P.; STAHL, A.; WARHOLM, M.; HOGBERG, J. Studies of the role of DNA fragmentation in selenium toxicity. Biochem. Pharmacol. 37: 3401-3406 (1988).

GOEGER, D.E. \& GANTHER, H. E. Oxidation of dimethyl selenide to dimethyl selenoxido by microssomes from rat liver and flavingcontaining monooxygenase from big liver. Arch. Biochem. Biophys. 310: 448-451 (1994).

GOLDMAN, I. \& KANTROWITZ, N. E. Cardiomyiopathy associated with slenium deficiency. N. Engl. J. Med. 305: 701 (1981).

GÜNZLER, W. A.; STEFFENS, G. J.; GROSSMANN, A.; KIM, S. M.; OTTING, F.; WENDEL, A., FLOHE, L. The amino-acid sequence of bovine glutathiione peroxidase. Hoppe seyelers Z Physiol Chem. 365(2): 195-212 (1984).

HAMILTON, A. Industrial poisons in the United States. New York, Macmillan Co. (1925).

HARTLEY, W. J. \& GRANT, A. B. A review of selenium-responsive diseases of New Zealand livestock. Fed. Proc. 20: 679-688 (1961).

HELZLSOUER, K.; JACOBS, R.; MORRIS, S. Acute selenium intoxication in the United States. Fed. Proc. Am. Soc. Exp. Biol. 44: 1670 (1985).

HOFFMAN, J. L. \& McCONNELL, K. P. Periodate-oxidized adenosine inhibits the formation of dimethylselenide and trimethylselenium ion in mice treated with selenite. Arch. Biochem. Biophys. 254(2): 534-540 (1986). 
HOJO, Y. Subject groups high and low in urinary selenium levels, workers exposed to heavy metals and patients with cancer and epilepsy. Bull. Environ. Contam. Toxicol. 26: 466-471 (1981).

HOLNES, D. L.; TARASCHUCK, I. G.; NETHERCOTT, J. R. Health status of cooper refiney workers with specific reference to selenium exposuro. Arch. Environ. Health. 44: 291-297 (1989).

HOPKINS, L. L.; POPE, A. L.; BAUMANN, C. A. Distribution of microgram quantities of selenium in tissues of the rat, and effects of previous selenium intake. J. Nutr. 88: 61-65 (1966).

HSIEH, H. S. \& GANTHER, H. E. Effects of stock or purified diet on rat liver enzymes involved in the synthesis of dimethyl selenide. J. Nutr. 106(11): 1577-1582 (1976).

HUNT, D.; LANE, H.; BEESINGER, D.; GALLGHER, K.; ROWLANDS, B. J.; JOHNSTON, D. Selenium and glutathione peroxidase levels in hospitalized burn patients. Fed. Proc. 42; 927 (1983).

KICE, J. L. \& LEE, W. S. Oxidation-reduction reactions of organoselenium compounds. 1. Mechanism of the reaction between seleninic acids and thiols. J. Am. Chem. Soc. 100(16): 5092-5102 (1977).

KITAHARA, J.; SEKO, Y.; IMURA, N. Possible involvement of active oxygen species in selenite toxicity in isolated rat hepatocytes. Arch. Toxicol. 67: 497-501 (1993).

KLAYMAN, D. L. IN: KLAYMAN, D. L.; GANTHER, W. H. (Eds.), Organic Selenium Compounds: Their Chemistry and Biology, John Wiley and sons, New York, 68-157pp (1973).

KUIKKA, J. \& NORDMAN, E. Measurement of (75Se) sodium selenite in the human body. Int. J. Nucl. Med. Biol. 5: 30-34 (1978).

LEAVANDER, O. A. \& BECK, M. A. Interacting nutritional and infectious etiologis of Keshan disease. Insights from coxsackie virus B-induced myocarditis in mice deficient selenium or vitamin E. Biol. Trace Elem. Res. 56: 5-21 (1997).

LEVANDER, O. A. \& BAUMANN, C. A. Selenium metabolism VI: Effects of arsenic on the excretion of selenium in bile. Toxicol. Appl. Pharmacol. 9: 106-115 (1966).

LINDER, M. C. Nutrition and metabolism of the trace elements. 7: 216-277 (1990).

MARTIN, J. L. \& GERLACK, M. Selenium metabolism in animals. Ann N Y Acad Sci. 192-193 (1972).

MARTIN, J. L. IN: KLAYMAN, D. L.; GANTHER, W. H. (Eds.), Organic Selenium Compounds: Their Chemistry and Biology, John Wiley and sons, New York, 663-691pp. (1973).

MOZIER, N. M.; McCONNELL, K. P.; HOFFMAN, J. L. S-adenosyl-Lmethionine: thioether S-methyltransferase, a new enzyme in sulfur and selenium metabolism. J. Biol. Chem. 263: 4527-4531 (1988). 
MUTH, O. H.; OLDFIELD, J. E.; REMMERT, L. F.; SCHUBERT, J. R. Effects of selenium and vitamin $E$ on white muscle disease. Science. 198: 1090 (1958).

OKAMOTO, Y. \& GÜNTHER, W. H. H. Organic selenium and tellurium chemistry. Annals of the New York Academy Sciences. 192: 181-192 (1972).

OLDFIELD, J.E. The two faces of selenium. J. Nutr 117: 2002-2008 (1987).

PAINTER, E. P \& FRANKE, K. W. Selenium in proteins from toxic foodstuff. II. The effect of acid hydrolysis. Cereal Chem. 13: 172-179 (1936).

PAINTER, E. P. The chemistry and toxicity of selenium compounds which special reference to the selenium problem. Chem. Rev 28: 179-213 (1941).

ROTRUCK, J.T.; POPE, A.L.; GANTHER, H.E.; SWANSON, A. B.; HAFEMAN, D. G.; HOESTRA, W. G. Selenium: Biochemical role as a component of glutatione peroxidase. Science. 179: 558-560 (1973).

SAITO, Y.; HASHIMOTO, T.; SASAKI, M.; HANAOKA, S.; SUGAI, K. Effect of selenium deficiency on cardiac function of individuals with severe disabilities under long-term tube feeding. Devel. Med. \& Child Neurol. 40: 743-748 (1998).

SCHONEICH, F. C.; NARAYANASWAMI, V.; ASMUS, K. D.; SIES, H. Reactitity ebselen and related selenorganic compounds with 1,2dichloro ethane radical cations and halogenated peroxyl radicals. Arch. Biochem. Biophys. 288: 18-25 (1990).

SCHWARZ, K. \& FOLTZ, C. M. Selenium as an integral part of factor 3 against dietary necrotic liver degeneration. J. Am. Chem. Soc. 79: 3292-3292 (1957).

SCHWARZ, K. \& PORTER, A. L. Some regularites in the structurefunction relationship of organoselenium compounds effective against dietary liver necrosis. Annals of the New York Academy of Sciences. 192: 200-214 (1972).

SEKO, Y.; SAITO, Y.; KITAHARA, J.; IMURA, N. Active oxygen generation by the reaction of selenite with reduced gluathione in vitro. In: Wendel, A (ed). Selenium in biology and medicine. Springer, Berlin Heldeiberg. New York. pp:70-73 (1989).

SHRIFT, A. \& VIRUPAKSHA, T. K. Seleno-amino acids in selenium containing accumulator plant. Biochim. Biophys. Acta. 100: 65-75 (1965).

SMITH, M. I.; FRANKE, K. W.; WESTFALL, B. B. The selenium problem in relation to public health. Public. Health. Rep. 51; 1496 (1936).

SPALLHOLZ, J. E. On the nature of selenium toxicity and carcinostatic activity. Free Radical Biology \& Medicine. 17: 45-64 (1993).

STADTMAN, T. C. Biosynthesis and function of selenocysteine-containing enzymes. J. Biol. Chem. 266: 16257-16260 (1991).

Rev. Ciência e Natura, Santa Maria, 22: 59 - 81 , 2000. 
STADTMAN, T. C. Selenium-dependent enzymes. Annu Rev Biochem. 49: 93-110 (1980).

SUNDE, R. A. \& EVENSON, J. K. Serine incorporation into the selenocysteine moiety of glutathione peroxidase. J. Biol. Chem. 262(2): 933-937 (1987).

THOMSON, C. D. \& STEWART, R. D. H. Metabolic studies of (75Se) selenomethionine and (75Se) selenite in the rat. Br. J. Nutr. 30: 139147 (1973).

THOMSON, C. D. \& STEWART, R. D. H. The metabolism of (75Se) selenite in young woman. Br. J. Nutr. 32: 47-57 (1974).

TRELEASE, S. F.; DISOMMA, A. A.; JACOBS, A.L. Seleno-amino acids found in Astragalus bisulcatus. Science. 132: 618 (1960).

TSEN, C.C. \& TAPPEL, A. L. Catalytic oxidation of glutathione and other sulphydril compounds biselenite. J. Biol. Chem 233: 1230-1232 (1958).

URSINI, F. \& BINDOLI, A. The role of selenium peroxidases in the protection against oxidative damage of membranes. Chemistry and Physics of lipids. 44: 255-276 (1987).

URSINI, F.; MAORINO, M.; VALENTE, M.; FERRI, K.; GREGOLIN, C. Purification of pig liver of a protein which protects lipossomes and biomembranes from peroxidative degradation and exhibits glutathione peroxidase activity on phosphatidylcholine hydroperoxidase. Biochem. Biophys. Acta. 710: 197-211 (1982).

VALENTINE, J. L.; KANG, H. K.; SPIVEY, G. H. Selenium levels in human blood, urine and hair in response to exposure via drinking water. Environ. Res. 17: 347-355 (1978).

WHANGER, P. D.; PEDERSEN, N. D.; HATFIELD, J.; WESWING, P. H. Absortion of selenite and selenomethionine from ligated digestive tract segments in rats. Proc. Soc. Exp. Biol. Med. 153: 295-297 (1976).

WILBER, C. G. Toxicology of selenium: A review. Clinical. toxicology. 17 (2): $171-230$ (1980).

XU, G. L.; WANG, S. C.; GU, B. Q.; YANG, Y. X.; SONG, H. B.; XUC,W. L.; LIANG, W. S.; ZHANG, P. Y. Further investigation on the role of selenium deficiency in the etiology and pathogenesis of Keshan disease. Biomed. Environ. Sci. 10: 316-326 (1997).

YAN, L. \& SPALLHOLZ, J. E. Free radicals generation by selenium compounds. Fase B. J. 5: 581 (1991).

YANG G.; WARG, S.; ZHOU, R. Endemic selenium intoxication of humans. Am. J. Clin. Nutr. 37: 872-881 (1983).

YANG, G. Q. Keshan disease: an endemic selenium-related deficiency disease. In: Trace Elements in Nutrition of Children. R. K. Chandra, Ed. New York, Raven Press, pp. 273-289 (1985).

YANG, G. Research on selenium-related problems in human health in China. In: Selenium in Biology and Medicine (Combs, G. F.; Spallholz, J. E.; Levander, O. A.; Oldfield, J. E., eds.), vol A, 9-32, Van Nostrand Reinhold, New York (1987).

80 Rev. Ciência e Natura, Santa Maria, 22: 59 - 81 , 2000. 
YASUDA, K.; WATANABE, H.; YAMAZAKI, S.; TODA, S. Gluthatione peroxidase activity of D, L-selenocystine and selenocystamine. Biochem. Biophys. Res. Commun. 98: 243-249 (1980).

YOUNG, V. R.; NAHAPETIAU, A.; JONGHORBONI, M. Selenium bioavailability with reference to human nutrition. American. J. Clinical. Nutrition. 35: 1076-1088 (1982).

ZIEGLER, D. M.; GRAF, P.; POULSEN, L. L.; STAHL, W.; SIES, H. NADPH-dependent oxidation of reduced ebselen, 2selenylbenzaniline, and 2(methylseleno)benzanilide catalized by pig flavin-containing monooxigenase. Chem. Res. Toxicol. 5: 163-166 (1991). 
Rev. Ciência e Natura, Santa Maria, 22: 59 - 81 , 2000. 